\title{
Dal Libro-Game alle storie digitali. Una didattica con Twine
}

\author{
di Doriana Dettole and Rosaria Leonardi
}

Ott 22, $2019 \mid \underline{\text { In evidenza, }}$ Pensare la didattica $\mid \underline{0 \mid}$

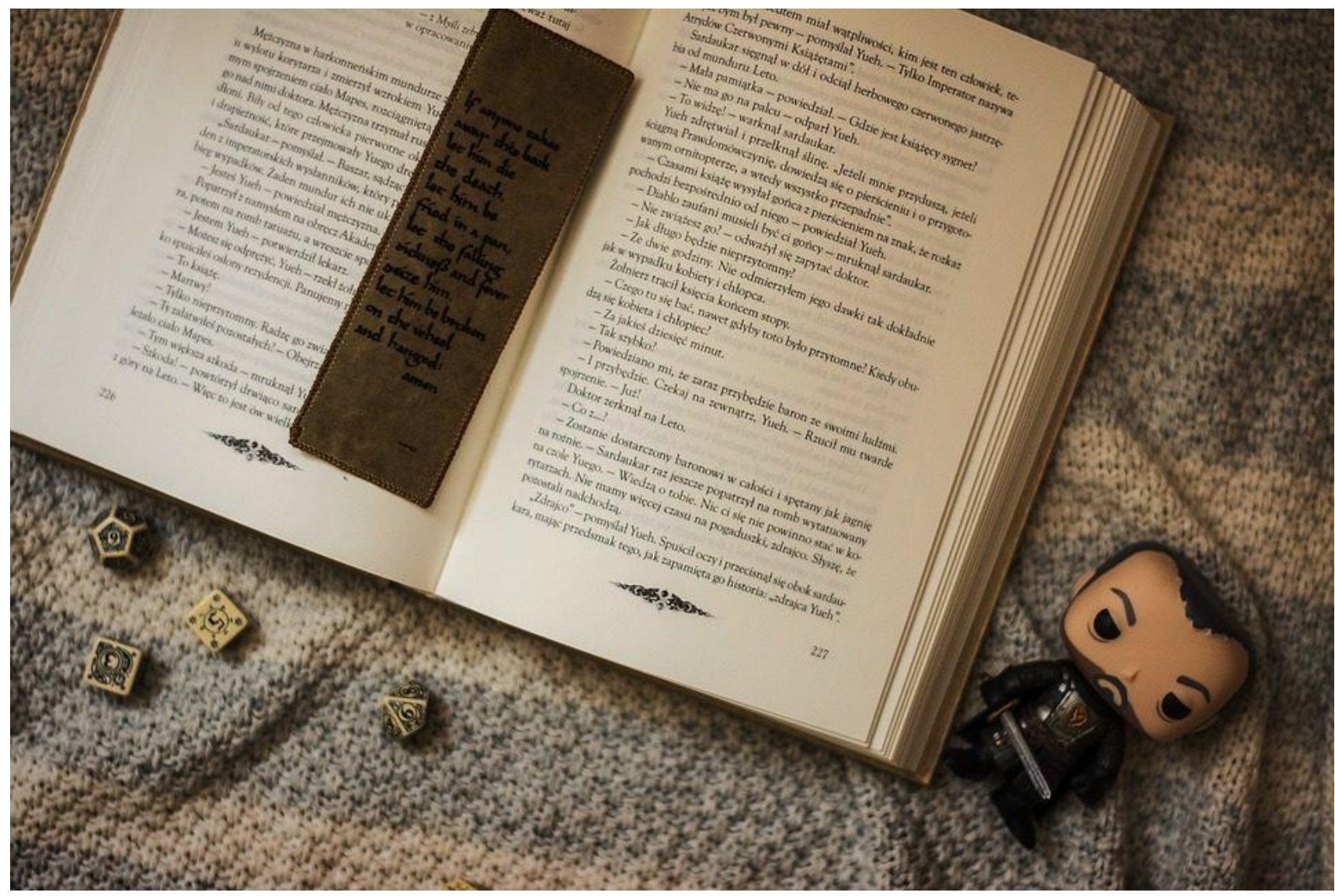

\section{Abstract}

Come venire incontro alle mutate caratteristiche di apprendimento delle nuove generazioni? In questo articolo si è cercato di tracciare la parabola che ha condotto il libro-game, nato come strumento ludico, verso applicazioni didattiche. I limiti del libro-game, in termini di giocabilità e di coinvolgimento dei giocatori, hanno, poi, stimolato la ricerca verso l'utilizzo di nuovi strumenti in ambito digitale.

La sperimentazione con Twine ha consentito la realizzazione di storie interattive a tema storico, come parte fondante di un laboratorio didattico sulla storia dell'integrazione europea. Si è, inoltre, provato a trasformare un testo scientifico di uno storico in un racconto digitale, nell'ipotesi di poter rendere fruibile, anche ai non specialisti, contributi accademici, spesso di difficile lettura e comprensione. Ciò in linea con le prospettive e le intenzioni della public history.

\section{INDICE}

- I libri-game: un po' di storia

- Costruire il libro-game

- Libri-game digitali. Dalle pagine del libro alle finestre dello schermo 
- L'uso didattico del libro game

- Sperimentazione sul tema delle migrazioni

- Sbrogliare la trama della complessità

- Bibliografia

- $\underline{\text { Sitografia }}$

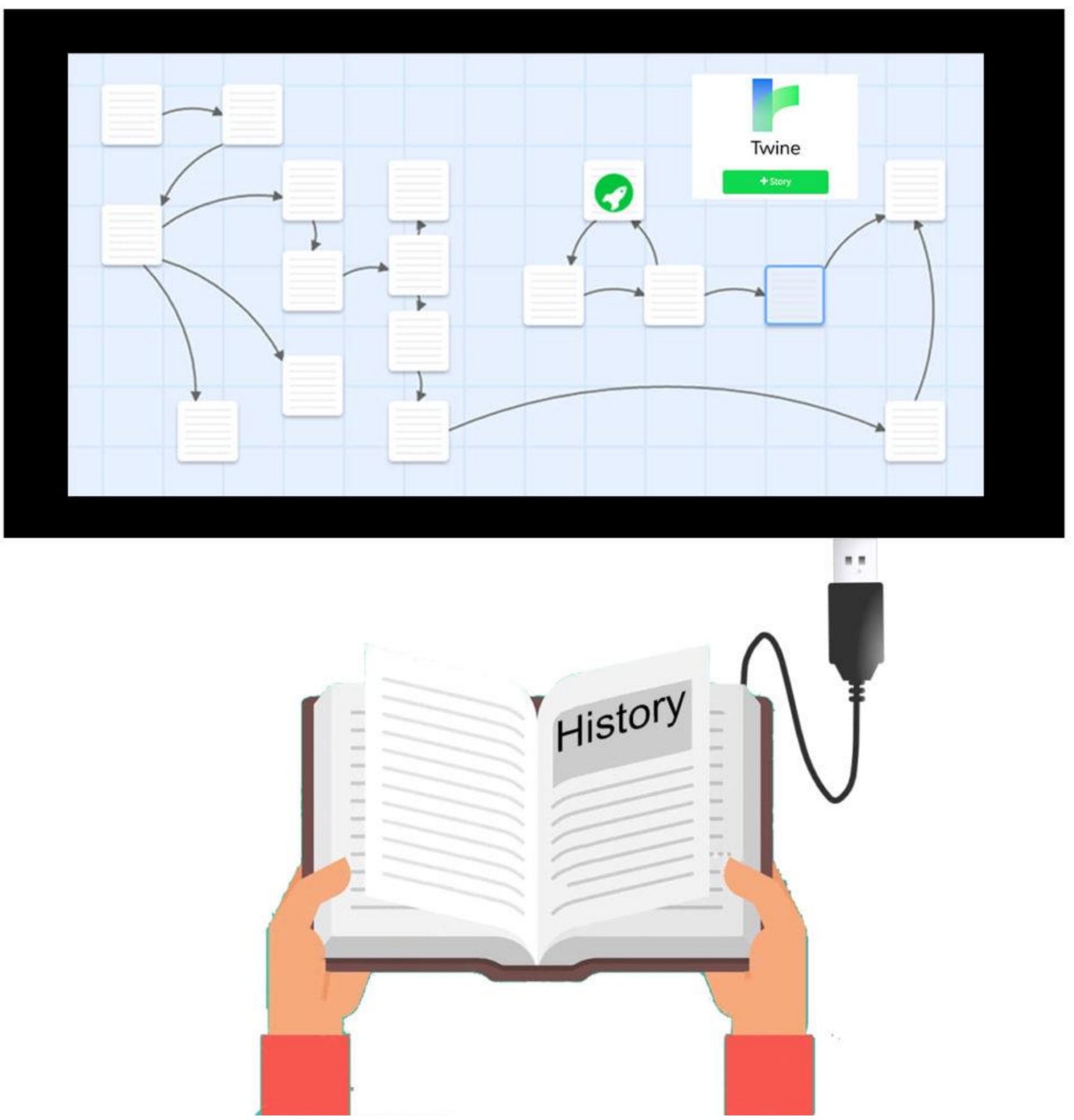

I libri-game: un po' di storia

A dare unità e originalità a una tipologia di testi di carattere ludico e letterario fu la casa editrice E.Elle di Trieste, che ha inventato e registrato il marchio «libro-game» con il quale ha poi 
pubblicato una collana di libri-gioco diretta da Giulio Lughi [Angiolino 2004a]. Il libro-game è approdato in Italia nel 1982 con la pubblicazione del volume di Edward Packard, Avventure nell'Isola. Si trattava di un racconto a bivi, destinato a bambini per la scuola primaria, scritto nel 1969 e pubblicato in Inghilterra nel 1976.

Si presentava come «una forma di racconto interattivo» [Angiolino 2004a, 15], strutturato in paragrafi e rimandi. L'autore, attraverso questa tipologia testuale, permetteva al lettore di scegliere i molteplici sviluppi della storia e, nel caso lo si volesse, di ritornare sui propri passi e di cambiare l'evoluzione del racconto. Per tale ragione questa tipologia di libro era costruita attorno al concetto di bivio: la scrittura, infatti, non seguiva un procedimento lineare e consequenziale ma si sviluppava seguendo percorsi paralleli, condizionati dalle scelte operate dal lettore. Tali scelte, poste in genere al termine dei singoli paragrafi, diventavano condizioni necessarie per il proseguimento della storia. Così strutturato, il racconto poteva essere definito "a bivi".

Fuori dai confini italiani le prime sperimentazioni di gamebook risalivano agli anni Sessanta con Raymond Quenau e la sua breve storia intitolata Un conte à votre façon, E. W. Hildick con Lucky Les: The adventures of a cat of five tales e Betty Orr-Nilsson con Denmystikapåsen [Angiolino 2004b].

In Italia, invece, a parte l'esperimento radiofonico di Gianni Rodari, il quale raccontava in diretta favole con più finali, fu dalla metà degli anni Ottanta che il modello del libro-gioco prese piede. La sua versatilità permise di raggiungere destinatari diversi per età e grado di scolarizzazione, di trovare spazio nell'editoria tradizionale, nella pubblicistica periodica e nella cinematografia e di essere utilizzato per scopi differenti: divulgativi, ludici, didattici.

\section{Costruire il libro-game}

Nella costruzione di un libro-game, la prima scelta da compiere riguarda l'alternativa tra componente ludica e narrativa e dunque la possibilità di dare maggiore o minor spazio all'uno o all'altro elemento. Quindi è necessario individuare il filone di appartenenza della storia (avventura, humor, fantasy, fantascienza, rosa, giallo, horror, storico) e delineare l'ambientazione e i caratteri dei personaggi (età, professione, ruolo all'interno delle vicende raccontate, storia pregressa).

Essenziale è il punto di vista: una storia narrata in prima persona permette al lettore di immedesimarsi con il protagonista del racconto e di essere emotivamente coinvolto così da condizionare anche il momento delle scelte; la terza persona, invece, consente un minore coinvolgimento emotivo da parte del lettore, una maggiore presenza dell'autore nel testo e l'instaurazione di un rapporto dialogico tra lettore e autore. La scelta tra prima e terza persona dipenderà dagli scopi per i quali si intende costruire il racconto.

La realizzazione del testo necessita, quindi, di un diagramma che abbia quali elementi costitutivi il numero dei paragrafi e i rimandi alle scelte da effettuare. Tale diagramma si rende indispensabile per tenere sotto controllo la struttura dell'intreccio, il quale presuppone l'individuazione di tutte le possibili scelte che il lettore può compiere e gli esiti e gli sviluppi che ne conseguono. 


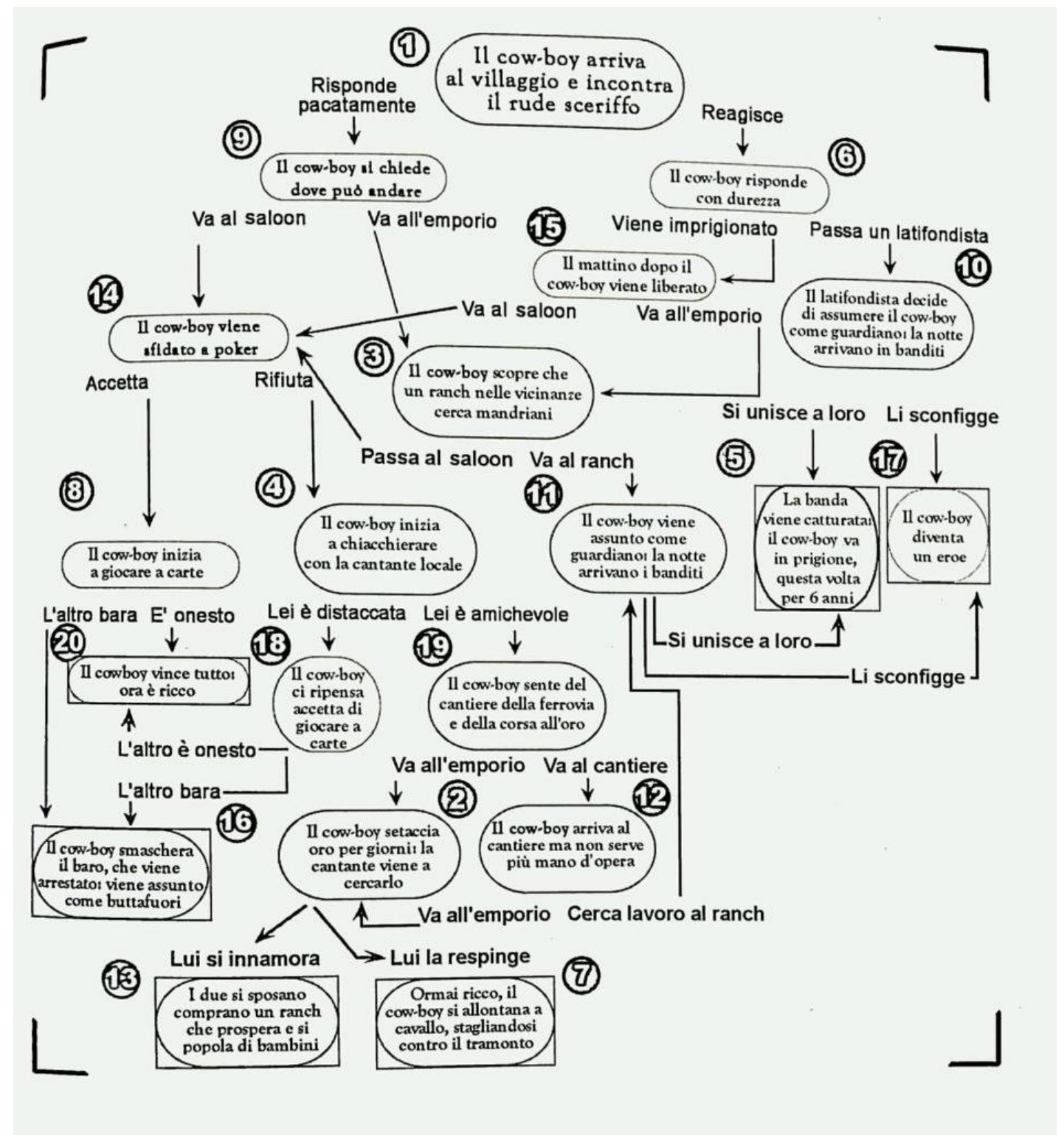

[Angiolino 2004a, p.115]

Come emerge dalla figura riportata, nella struttura sono presenti anche paragrafi che non necessitano di alcuna scelta. Si tratta di uno strumento narrativo utile a evitare il corto circuito della storia: troppe scelte, infatti, potrebbero portare all'abbandono della lettura. Allo stesso modo, un'eccessiva presenza di paragrafi lineari e privi di bivi potrebbe determinare una condizione di noia e conseguente rinuncia all'attività.

Nel racconto a bivi è possibile anche inserire attività supplementari per scopo ludico o didattico (schede profilo del personaggio, risorse come oggetti e incantesimi da utilizzare nel corso del racconto, dadi da lanciare, appunti da prendere, mappe, immagini, indizi ed enigmi, attività di scrittura, indovinelli), soprattutto nel caso in cui si prediliga un'impostazione soggettiva della storia.

Libri-game digitali. dalle pagine del libro alle finestre dello schermo

La costruzione e l'utilizzo del libro gioco, pur presentando numerosi aspetti positivi dal punto di vista ludico-didattico, soffre, altresì, di limiti concernenti la dimensione pratica dell'attività. Il 
formato libro cartaceo rende difficoltoso sia il momento della realizzazione del racconto "a bivi" sia la giocabilità, che potrebbe risultare farraginosa e poco agevole.

Il periodo storico nel quale si è diffusa questa tipologia testuale non consentiva esiti differenti. Lo sviluppo delle tecnologie informatiche che ha inaugurato la nostra era digitale ha permesso, invece, fin dai primi anni Novanta, di pensare al racconto "a bivi" in un linguaggio differente: quello ipertestuale.

Il primo esperimento, effettuato da Enrico Colombini e Chiara Tovena, risalente al 1988, permise la trasformazione del libro gioco In cerca di fortuna in un ipertesto per Mac. Pochi anni dopo lo stesso Colombini progettò il software open source Idra [www.erix.it/idra.html] con il quale era possibile creare libri gioco in linguaggio html. [Angiolino 2004a].

Il libro-game digitale, oltre ad essere più agevole da realizzare, offre una maggiore facilità di gioco e garantisce una divulgazione più vasta, soprattutto nell'era dei "nativi digitali", ovvero di coloro che, nati in un'epoca di profonde trasformazioni tecnologiche, hanno un modo di comunicare e apprendere profondamente diverso rispetto a quello delle generazioni precedenti.

Dalla realizzazione di Idra, si è assistito alla diffusione di numerosi e sempre più complessi programmi di questo genere. Una semplice ricognizione su un qualsiasi motore di ricerca dà conto della quantità e della varietà di opzioni a disposizione per la realizzazione di gamebooks.

Tra questi, Twine risulta essere uno dei più diffusi e utilizzati per la sua struttura, accessibilità e perché dispone di un manuale completo e di un forum tramite cui è possibile confrontarsi con gli altri utenti. Si tratta di un software open source, ideato da Chris Klimas nel 2009 per la creazione di narrativa interattiva [twinery.org], che trasferisce in ambiente digitale la struttura del libro gioco, per mezzo di specifici codici previsti dal programma. 


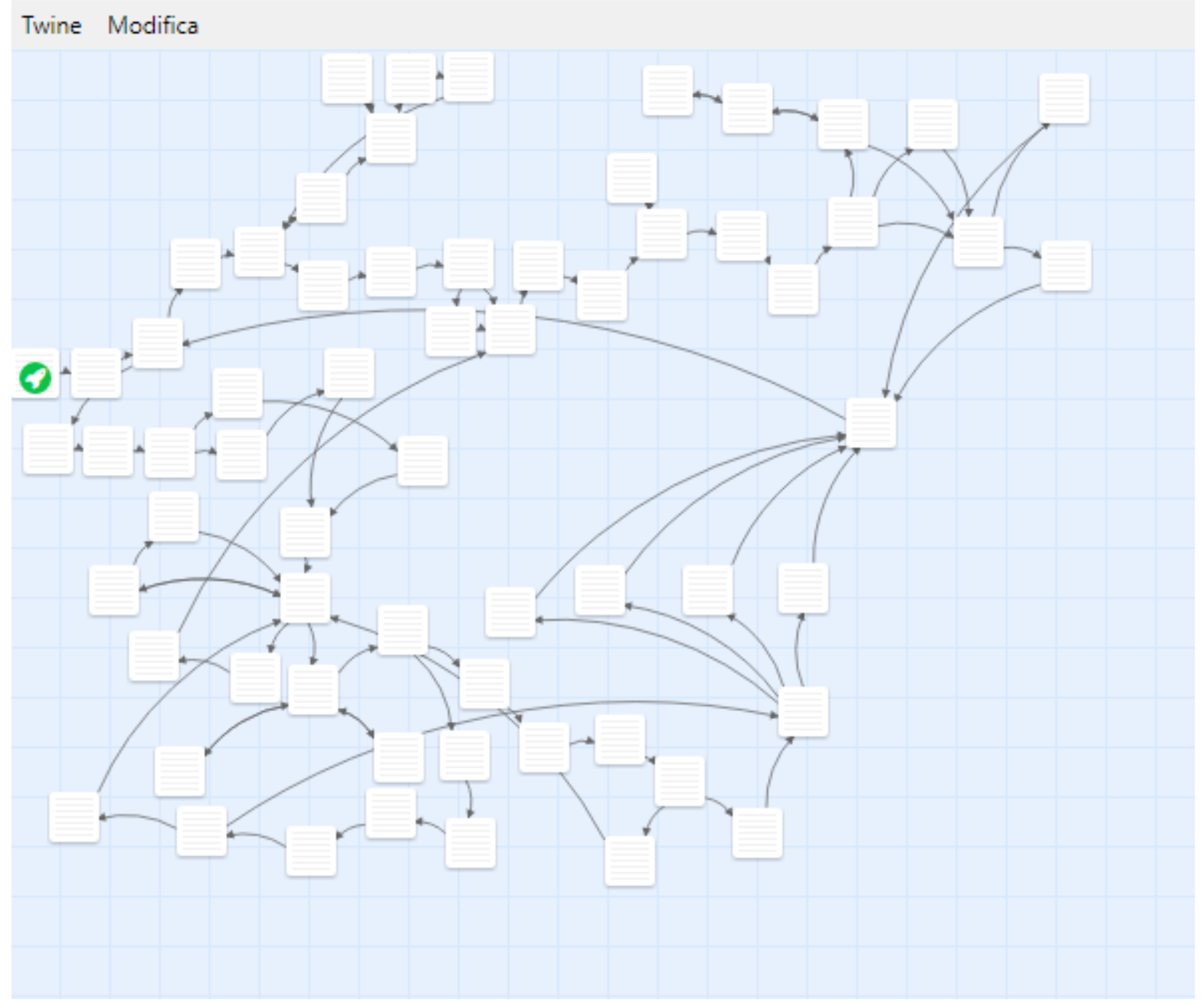

Come si evince dall'immagine sopra riportata, l'organigramma è del tutto simile a quello che abbiamo presentato nel paragrafo precedente. Le pagine del libro si trasformano in schede, ognuna delle quali rappresenta un paragrafo, mentre i rimandi si traducono in collegamenti ipertestuali. All'interno della storia è possibile aggiungere elementi multimediali quali immagini, suoni, Google maps e video. L'esito è un elaborato in linguaggio html, facilmente accessibile da qualsiasi dispositivo elettronico.

\section{L'uso didattico del libro-game}

Le potenzialità del libro-game, insomma, sono spendibili anche in ambito didattico, soprattutto in quello della didattica della storia. In Italia, gamebooks di tipo storico sono stati realizzati fin dalla metà degli anni Novanta - Il gobbo maledetto, Spartaco. La rivolta dei gladiatori, I misteri delle catacombe [Angiolino e Alegy 1993; Zotti 1995; Angiolino, Di Giorgio e Garello 2000] -, ma si trattava, perlopiù, di giochi non progettati esplicitamente per la scuola.

Magna Grecia, invece, rappresenta un esperimento di racconto "a bivi” realizzato per fini didattici.

È un libro gioco ambientato nella Grecia dell'VIII sec. a.C., nel quale il giocatore, immedesimandosi con un gruppo di coloni di Corinto, deve compiere scelte storicamente rilevanti per fondare una nuova colonia. [Brusa e Bresil 1994]. 
Si tratta di un approccio didattico democratico e collettivo nel quale la conoscenza storica non è veicolata unidirezionalmente dal docente, ma scaturisce dall'esperienza di gioco e dal continuo confronto tra gli alunni e tra questi e l'insegnante. A quest'ultimo è riservato un ruolo fondamentale, quello di organizzare e coordinare il momento del gioco e quello successivo del debriefing: grazie al libro-game, infatti, è possibile accogliere ogni proposta da parte degli studenti facendola diventare momento di confronto, approfondimento e scambio di conoscenze.

Il debriefing è parte integrante dell'uso didattico del libro-game, l'occasione nella quale gli studenti possono riflettere in modo critico sull'esperienza di gioco e ripercorrerne le dinamiche del processo storico. Al debriefing possono seguire molteplici attività didattiche: dall'historytelling al debate, all'uso delle fonti, che permettono lo sviluppo delle competenze disciplinari e trasversali.

Con il libro digitale si viene incontro ai bisogni degli alunni del nuovo millennio, che, cresciuti con il computer, «pensano diversamente dal resto di noi: sviluppano menti ipertestuali parallele, non sequenziali» [Salvatore 2019]. Anche Paolo Ferri si attesta su posizioni analoghe quando afferma che «l'apprendimento risulta essere non più centralizzato ma frammentato, improntato ad altre attività che non potrebbero essere contemplate in un modello didattico di stampo classico. L'uso delle nuove tecnologie permette di aumentare le capacità e le prestazioni. Il libro perde la priorità assoluta e l'esclusività» [Ferri 2011].

In realtà, il dibattito su questi temi è ancora aperto. Perciò, le potenzialità delle tecnologie digitali e della mente ipertestuale come si collocano rispetto alla forma-libro? Sono inconciliabili o è possibile trovare un modo per renderle complementari e funzionali allo stesso tempo?

Il libro digitale dimostra come tale conciliabilità sia realizzabile. Anzi, la flessibilità consentita dalle nuove tecnologie può potenziare la didattica in diverse direzioni (per esempio la didattica del cooperative learning e del problem solving), attivando diverse competenze perfettamente in linea con i fini delle nuove metodologie dell'era dei "nativi digitali".

\section{Sperimentazione sul tema delle migrazioni}

Nell'ambito della Cattedra Jean Monnet - Hicom 2018-21 Sfide storiche e politiche della memoria dell'integrazione europea. Mezzogiorno e Mediterraneo - dell'Università degli Studi di Bari, si è avuto modo di sperimentare, attraverso la realizzazione di un laboratorio ludico-didattico chiamato From Europe/to Europe. Storie di frontiere e migrazioni, le potenzialità del libro gioco digitale affrontando il tema del rapporto tra Europa e migrazioni nel lungo periodo, dalla fine dell' 800 all'inizio del nuovo millennio.

Abbiamo realizzato, servendoci di Twine, quattro storie digitali relative a quattro nuclei migratori: l'emigrazione italiana verso le Americhe tra fine ' 800 e inizi del '900; 1'emigrazione italiana verso il Nord Europa alla fine della II Guerra Mondiale; l'emigrazione rumena verso l'Europa Occidentale post '89; 1'emigrazione africana attraverso il Mediterraneo.

$\mathrm{Al}$ centro di ogni storia uno o più protagonisti narrano in prima persona le proprie esperienze di viaggio e migrazione determinando, soprattutto al momento delle scelte, un coinvolgimento totale con il giocatore. A rendere ancor più avvincenti e interattive le narrazioni sono i contenuti multimediali quali immagini, suoni, video e Google maps.

La costruzione dei racconti digitali si è basata sullo studio delle fonti documentali, le quali hanno da una parte fornito episodi fedelmente riportati nelle storie, dall'altra, invece, hanno ispirato vicende liberamente modellate alla narrazione. 
Pur trattando di tempi così diversi e lontani tra loro, le storie sono state pensate per ricondurre a un elemento comune. La storia europea di lungo periodo può essere, infatti, letta come una storia di frontiere che si allargano e si restringono, si chiudono, si aprono e si modificano. Per questo motivo il concetto di frontiera è stato il filo conduttore delle storie ed elemento essenziale per una riflessione sul tempo presente.

Si è, quindi, pensato al debriefing sia come momento di condivisione delle esperienze migratorie e di gioco sia come strumento di approfondimento sul rapporto tra Europa e migrazioni di ieri e di oggi. Agli insegnanti spetta il ruolo di mediare e coordinare le attività di gioco e di dibattito; a tal fine sono forniti loro strumenti documentali e bibliografici, nonché uno schema di supporto alla discussione. Inoltre, sono offerte una serie di proposte didattiche post debriefing volte ad approfondire i temi emersi durante il dibattito. Si tratta di attività volte ad avvicinare gli studenti al lavoro sulle fonti, a comprendere le conoscenze sul tema delle migrazioni, a scrivere dell'argomento trattato e, infine, a sperimentare un differente metodo di indagine quale quello dell'intervista.

Sul sito della Cattedra Jean Monnet dell'Università degli Studi di Bari è possibile accedere ai contenuti del suddetto laboratorio che è stato oggetto di un corso di formazione e aggiornamento per docenti - L'Europa nel mondo contemporaneo e i dilemmi del presente. Per una didattica dell'UE e della cittadinanza europea - e di una prima sperimentazione didattica, con studenti delle scuole secondarie di primo e secondo grado, durante il Convegno Internazionale Play with time/Tempo in gioco. Historia e Philosophia Ludens, tenutosi a Bari dal 15 al 17 aprile 2019.

\section{Sbrogliare la trama della complessità}

Perché non utilizzare questi strumenti per avvicinare i non specialisti al lavoro degli storici, condurli verso i documenti, i risultati aggiornati, le pratiche della ricerca?

Un esperimento in tal senso ha riguardato la trasformazione del testo di Francesco Altamura Dalle Dolomiti alle Murge, profughi trentini della Grande Guerra. Storie e memorie delle popolazioni di Primiero e Vanoi sfollate in Puglia nel 1916 [Altamura, 2017] in una storia digitale, intitolata Quei profughi venuti dal nord. 


\section{Capitolo II}

\section{Il viaggio}

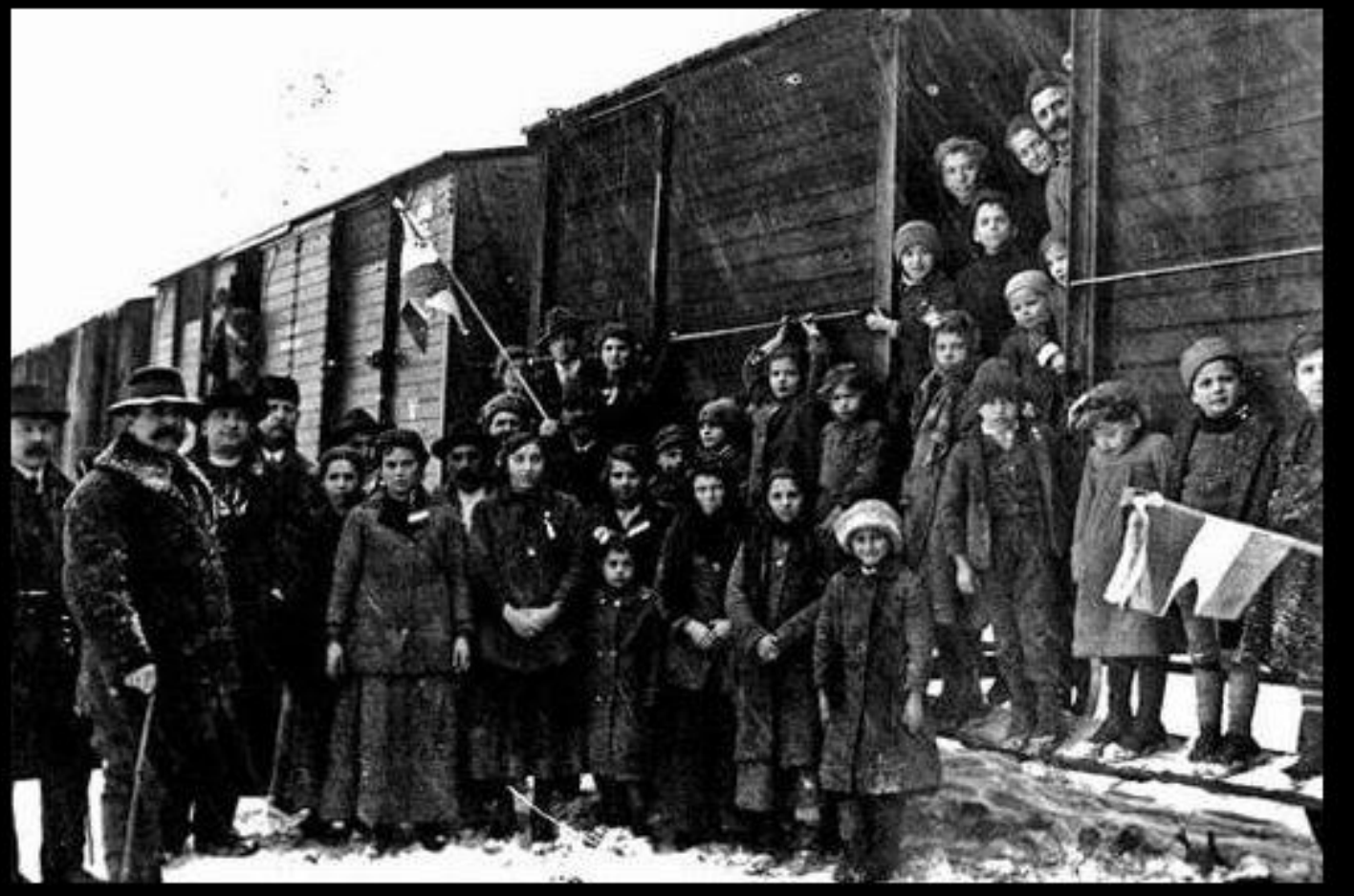

Il volume fa luce sulle vicende delle popolazioni di Primiero e Vanoi, costrette a lasciare il Trentino nel 1916 e condotte profughe in Puglia; sulla macchina dell'accoglienza che per la prima volta il Regno d'Italia e i comuni dovettero assemblare; sulla difficoltà ad adattarsi al profugato e sui rapporti tra profughi e popolazioni locali.

Si è cercato di ricondurre le vicende richiamate dall'autore Altamura a una dimensione intima e personale. Si è scelto di narrare in prima persona, seguendo il punto di vista della profuga Gilda Zortea. Ciò ha permesso di rendere più reali e ravvicinati gli avvenimenti e gli snodi storici contenuti nel testo. È la dimostrazione di come una stessa storia possa avere esiti narrativi differenti. 


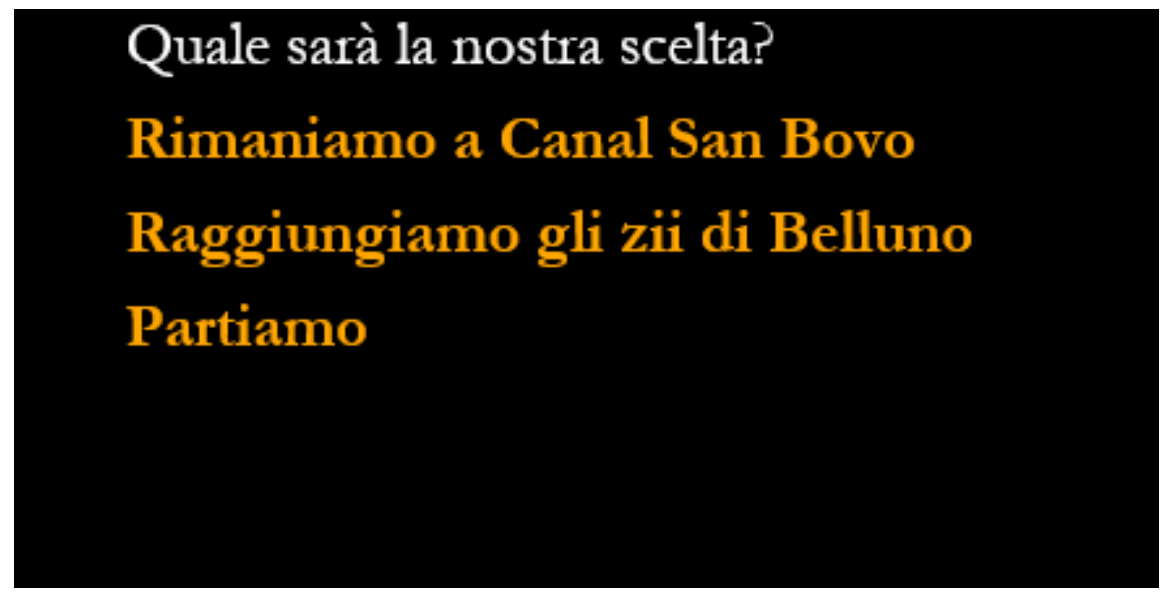

Tuttavia, nella rielaborazione del volume, nonostante l'introduzione di bivi e scelte, abbiamo fedelmente rispettato la veridicità dei fatti, eliminando quasi del tutto la componente creativa. Ciò non ha però inficiato la giocabilità della storia, la quale si presta anche alla creazione di laboratori didattici o all'approfondimento, sottoforma di contenuti digitali, degli argomenti dei manuali scolastici.

Quello presentato è un esempio che dimostra, inoltre, come i racconti "a bivi" digitali permettano di rendere i testi accademici, considerati dai non addetti ai lavori di difficile lettura e comprensione, più chiari e adatti a un pubblico più vasto, in linea con le intenzioni e le prospettive della public history.

$\underline{\text { Leggi e gioca a "Quei profughi venuti dal Nord" }}$

\section{Bibliografia}

- Altamura Francesco 2017, Dalle Dolomiti alle Murge, profughi trentini della Grande Guerra. Storie e memorie delle popolazioni di Primiero e Vanoi sfollate in Puglia nel 1916, Bari: Edizioni dal Sud

- Angiolino Andrea 2004a, Costruire i libri-gioco. Come scriverli e utilizzarli per la didattica, la scrittura collettiva e il teatro interattivo, Casale Monferrato: Edizioni Sonola.

- Angiolino Andrea 2004b, Narrare per bivi, rill.it

- Angiolino Andrea, Alegi Gregory 1993, Il gobbo maledetto, Novecento GeC

- Angiolino Andrea, Domenico Di Giorgio, Garello Francesca 2000, I misteri delle catacombe, Elledici

- Brusa Antonio, Bresil Luciana 1994, Il laboratorio delle simulazioni: Magna Grecia. Gioco di Storia e geografia per la scuola media, liberamente ispirato a Braudel, e forse anche a Erodoto, in Bresil Luciana, Brusa Antonio, Laboratorio, vol. 1, Milano: Ed. Scol. Bruno Mondadori

- Ferri Paolo, Nativi digitali, Bruno Mondadori, Milano, 2011.

- Salvatore Francesca 2019, Game History: innovazione didattica tra gioco e realtà, in "Sapere pedagogico e pratiche educative", 3

- Zotti Nicola 1995, La rivolta dei gladiatori, Quality Game

Sitografia

- https://gamebooks.org

- http://librogame.net

- https://twinery.org 
- http://www.erix.it/idra.html 